\title{
Mixed convection in a channel partially filled with metal foam blocks
}

\author{
Syrine Khadhrawi ${ }^{1,{ }^{*}}$, Fakhreddine Segni Oueslati ${ }^{2,}$, and Rachid Bennacer ${ }^{3}$ \\ ${ }^{1}$ Faculté des Sciences de Tunis (FST), Dépt. de Physique, LETTM, Campus Universitaire El-Manar, 2092 El Manar, Tunisia. \\ ${ }^{2}$ Ecole Nationale d'Ingénieurs de Carthage (ENICarthage), 45 rue des entrepreneurs, Charguia II, Tunis Carthage 2035 Tunisia. \\ ${ }^{3}$ LMT, ENS Paris-Saclay, CNRS, Université Paris-Saclay, 94235 Cachan, France.
}

\begin{abstract}
The present work is a numerical simulation of the mixed convection of an incompressible fluid in a horizontal channel under sun radiation partially filled with metal foam blocks. The Darcy-Brinkman model is adopted. The control volume approach is used to solve the motion and energy equations governing mixed convection. The study focuses on the effect of certain control parameters such as the Rayleigh number $(\mathrm{Ra})$, the thermal conductivity ratio and the porosity on the flow structure and heat transfer. Indeed, the results for flow structures and temperature distribution are presented in the form of velocity fields with streamtraces and isotherms, while the average Nusselt number $(\mathrm{Nu})$ is used to quantify heat transfer.
\end{abstract}

\section{Introduction}

The problem of mixed convection in porous structures has been the subject of many scientific and industrial problems such as geophysics, heat exchangers, hydrology, chemical processes and biology. Also, there are several applications of this phenomenon such as the storage of nuclear waste and solar collectors. This interest is due to the fact that this kind of structure improves the heat transfer performance, reduces the boundary layer thickness, increases the surface area in contact with the fluid, intensifies the mixing of the flowing fluid and thereby enhances the convection heat transfer. In the literature several articles have numerically treated convection in a partially porous cavity, the state of the art has been very well summarized in the monographs by Nield and Bejan [1] and Vafai [2]. Although research in porous media is not new, Kaviany [3] began work more than 150 years ago, but problems with high-porosity cellular materials only started in the last 20 years. Metal foams as a relatively new class of porous media are a network of randomly oriented ligaments, consisting of connected void namely pores with nearly uniform size and shape, the most commercially available metal foams are based on aluminum, copper, nickel and metal alloys. The foregoing literature review shows most of the numerical, experimental and analytical studies of flow and heat transfer in metal foams focused either on natural, forced or mixed convection. A numerical study of natural convection in a cubic cavity partially filled with a porous medium made by F. Habbachi et al. [4]. The authors found that fluid flow and heat transfer are controlled by the thickness of the porous matrix and the thermal conductivity ratio. A.kacem et al. [5] published a numerical study of natural convection in a porous cubic cavity. The work reported that the increase of the Darcy number and the Rayleigh number porous leads to the intensification of the flow and the heat transfer is mainly convective.

W.Lu et al. [6] have developed analytically forced convection heat transfer solutions in a parallel plate channel partially filled with metal foam. Their results show that the pressure decrease in a partially filled foam channel is lower than that of a totally filled channel. The authors have deduced that if the amount of fluid entering the foam is large, the fluid is enhanced by the foams and thereafter results in improved heat transfer performance and high flow resistance. Other researchers analyzed numerically the natural convection in horizontal cavity partially filled with aluminum foam and heated from above B.Buonomo et al. [7]. The authors concluded that the presence of metal foam with low values of PPI, the heat transfer is improved. The same authors [8] examined experimentally and numerically mixed convection in a cavity heated from below. It has been noticed that the presence of the metal foam at the level of the channel causes a decrease of the temperature for weak PPI at the entrance of the heated channel. The flow behavior of the fluid in a partially filled channel of the metal foam block is examined experimentally by F.S.Anuar et al. [9]. They have noticed that foams with low pore densities facilitate flow in the porous matrix, and the blocking rate does affect temperature distributions. X.Bai et al. [10] studied numerically the heat transfer by forced convection in a cavity filled with a metal foam. Their results show that the existence of the functional caliber metal foam in the channel causes the increase of the heat transfer coefficient as well as the increase of the pressure drop.

\footnotetext{
*Corresponding author: khadhrawi.sirine@gmail.com * fakhri.oueslati@gmail.com
} 
The main goal of this paper is a numerical study $3 \mathrm{D}$ of mixed convection in a channel, under sun radiation, partially filled with metal foam block in out of local thermal equilibrium. Our numerical simulation gave rise to several results relating to the control parameters of our problem. Thus, the effect of these parameters on the flow structures and the heat transfer was invested.

\section{Analysis and Modeling}

In this paper, it is proposed to study the phenomenon of mixed convection in a horizontal channel partially filled with metal foam out of local thermal equilibrium with the fluid. The geometry of the physical problem studied is presented in Figure 1. The cavity is of length $\left(\mathrm{L}^{\prime}=6\right)$, height and depth $\left(\mathrm{H}^{\prime}=1\right)$. The speed and the inlet temperature are kept constant $\left(\mathrm{V}_{0}\right.$ and $\left.\mathrm{T}_{0}\right)$. In addition, the lower and upper walls are subjected to a uniform temperature $\left(\mathrm{T}_{\mathrm{w}}^{\prime}\right)$ on the porous matrix at the top and bottom of the fluid layers (bottom), while the other walls are adiabatic. Among the simplifying hypotheses used to facilitate the resolution of conservation equations are: The fluid saturating the porous medium is Newtonian and incompressible, the porous medium is homogeneous isotropic saturated with a fluid (air), the flow is laminar, the transfer of radiation heat is negligible. In addition, the boussinesq approximation is adopted :

$$
\rho=\rho_{0}\left(1-\beta_{T} \Delta T^{\prime}\right)
$$

with

$$
\beta_{T}=-1 / \rho_{0}\left(\partial \rho / \partial T^{\prime}\right)_{P}
$$

\section{Solar radiation}

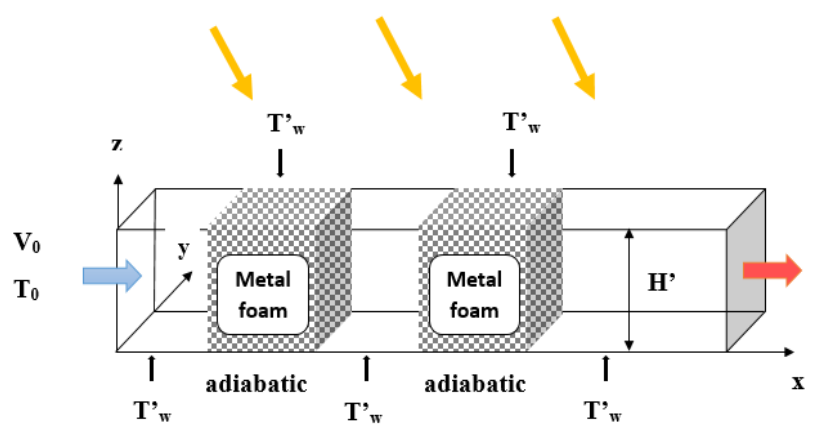

Fig. 1. Physical domain considered and coordinates system

In the present study, the solution of our problem is retained by solving the Navier-Stokes equations in the fluid domain and the flow is modeled by utilizing the Darcy-Brinkman equations for the porous layer. Retaining the corresponding terms in the conservation equations leads to the full Navier-Stokes equation in the fluid or the Darcy-Brinkman model in the porous media. This implies numerical difficulties in terms of convergence and accuracy at the fluid-porous interface when the permeability contrast is high (low Darcy number in the porous layer). The following dimensionless variables are used:

$$
\left\{\begin{array}{l}
(x, y, z)=\left(x^{\prime}, y^{\prime}, z^{\prime}\right) / H^{\prime} ;(u, v, w)=\left(u^{\prime}, v^{\prime}, w^{\prime}\right) / V_{0} \\
P=\frac{P^{\prime}}{P_{0}}=\frac{P^{\prime}}{\rho V_{0}^{2}} \text { with } P_{0}=\rho V_{0}^{2} ; t=\frac{t^{\prime}}{t_{0}}=\frac{t^{\prime} V_{0}}{H^{\prime}} \\
t_{0}=\frac{H^{\prime}}{V_{0}} ; T=\frac{\left(T^{\prime}-T_{0}^{\prime}\right)}{\Delta T^{\prime}} ; T_{0}^{\prime}=T_{(0,0)}^{\prime} ; \Delta T^{\prime}=T_{w}^{\prime}-T_{0}^{\prime}
\end{array}\right\}
$$

In addition, the Darcy-Brinkman model was adopted in this analysis. By introducing the adimensional quantities given above into the conservation equations, we obtain another system of governing equations in dimensionless form:

$$
\begin{gathered}
\vec{\nabla} \cdot \vec{V}=0 \\
\frac{1}{\varepsilon} \frac{\partial \vec{V}}{\partial t}+\frac{1}{\varepsilon^{2}}(\vec{V} \cdot \vec{\nabla}) \vec{V}=-\vec{\nabla} P+\frac{1}{\operatorname{Re}}\left(-\frac{1}{D a} \vec{V}+\nabla^{2} \vec{V}+\frac{R a}{\operatorname{Pr} \times \operatorname{Re}} \cdot T \cdot \vec{k}\right) \\
\left(\frac{\partial T_{s}}{\partial t}\right)=\frac{\tilde{\alpha}}{\operatorname{Pr} \operatorname{Re}}\left\{\nabla^{2} T_{s}+R_{\lambda} B i\left[T_{f}-T_{s}\right]\right\} \\
\left(\frac{\partial T_{f}}{\partial t}+\vec{V} \cdot \vec{\nabla} T_{f}\right)=\frac{1}{\operatorname{Pr} \operatorname{Re}}\left\{\nabla^{2} T_{f}+B i\left[T_{s}-T_{f}\right]\right\}
\end{gathered}
$$

Foregoing equations introduce the following dimensionless parameters:

The Prandtl number $\operatorname{Pr}=v / \alpha$,

The Darcy number $D a=K / H^{\prime}$,

The Rayleigh number $\mathrm{Ra}=g \beta_{T} \Delta T H^{\prime 3} / v \alpha$,

The Biot number $B i=h_{s f} a_{s f} H^{\prime 2} /\left(\varepsilon \lambda_{f}\right)$

The thermal conductivity ratio $R_{\lambda}=\varepsilon \lambda_{f} /\left((1-\varepsilon) \lambda_{s}\right)$

The thermal diffusivity ratio $\tilde{\alpha}=\alpha_{s} / \alpha_{f}$

The boundary conditions for the governing equations are the non-slip condition on all the walls of the cavity even though. The lower and upper walls are subjected to a uniform temperature $\left(\mathrm{T}^{\prime}{ }_{\mathrm{w}}\right)$ on the porous matrix at the top and bottom of the fluid layers (bottom), while the other walls are adiabatic. The dimensionless boundary conditions can be mathematically expressed as follows:

$$
\begin{aligned}
& x=0 \quad 0 \leq y \leq 1 \quad 0 \leq z \leq 1 \quad \Rightarrow T=0, u=1 \\
& x=6 \quad 0 \leq y \leq 1 \quad 0 \leq z \leq 1 \quad \Rightarrow \frac{\partial T}{\partial x}=\frac{\partial u}{\partial x}=\frac{\partial v}{\partial x}=\frac{\partial w}{\partial x}=0 \\
& y=0 ; 1 \quad 0 \leq x \leq 6 \quad 0 \leq z \leq 1 \quad \Rightarrow \frac{\partial T}{\partial y}=v=0 \\
& z=0 \quad 0 \leq x \leq 6 \quad 0 \leq y \leq 1 \Rightarrow\left\{\begin{array}{cc}
\delta=0 & T=1 \quad w=0 \\
\delta=1 & \frac{\partial T}{\partial z}=w=0
\end{array}\right\} \\
& z=1 \quad 0 \leq x \leq 6 \quad 0 \leq y \leq 1 \Rightarrow\left\{\begin{array}{cc}
\delta=0 & \frac{\partial T}{\partial z}=w=0 \\
\delta=1 & T=1 \quad w=0
\end{array}\right\}
\end{aligned}
$$

With $\delta=\left\{\begin{array}{l}1: \text { milieu poreux } \\ 0: \text { milieu fluide }\end{array}\right\}$ 
The results for the total heat transfer rate across the enclosure will be presented in terms of the Nusselt number defined as:

$$
\begin{gathered}
N u=\frac{\delta(\tilde{\lambda}-1)+1}{1-T_{\text {moy }}} \frac{\partial T}{\partial z} \\
T_{\text {moy }}=\frac{\iint T \cdot u \cdot d x d y}{\int u d x}
\end{gathered}
$$

The numerical resolution was performed by a finite volume method-based calculation code described in Patankar [11] for the purpose of solving the dimensionless equations (3-6) governing our problem. The resulting algebraic equations were solved line by line using the SIMPLE algorithm. We also used iterative methods coupled with a $122 * 42 * 42$ nodes multi-grid acceleration technique for more accurate results. The convergence criteria are the conservation of mass, momentum and energy mean, as well as the convergence of preselected dependent variables with a prescribed tolerance of $10^{-6}$

\section{Results and discussion}

In this part, we will focus on studying the effect of porosity $(\varepsilon)$, the Rayleigh number $(\mathrm{Ra})$ and the thermal conductivity ratio $(\tilde{\lambda})$ considering that the metal foam and the fluid are out of local thermal equilibrium.

\subsection{Effect of porosity}

Isotherms, velocity fields and streamtraces for different porosities $(\varepsilon), \operatorname{Re}=50, \mathrm{Ra}=10^{4},(\tilde{\lambda}=1)$ and $\mathrm{Da}=10^{-3}$ are shown in Figure 2. There is a similarity of the isotherms for different porosities.
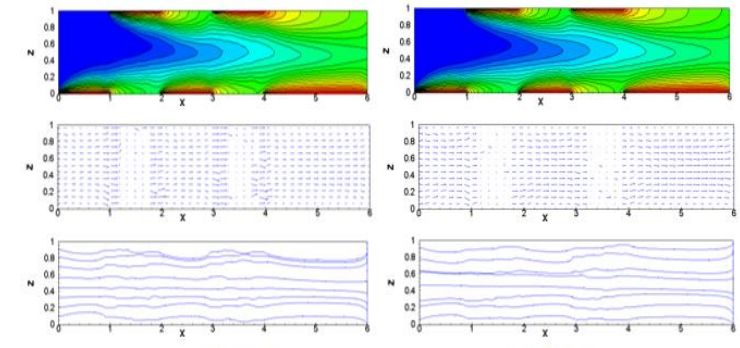

$\varepsilon=0.882$

$\varepsilon=0.901$
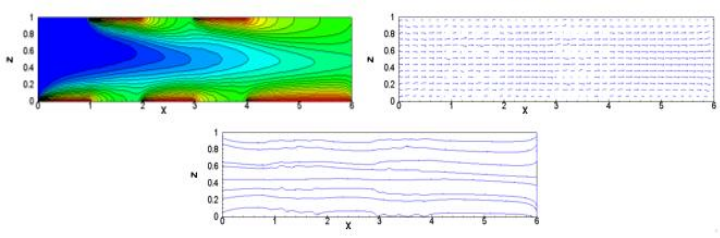

$$
\varepsilon=0.97
$$

Fig. 2. Isotherms, velocity fields and streamtraces for different porosities, $\operatorname{Re}=50, \operatorname{Ra}=10^{4},(\tilde{\lambda}=1)$ and $\mathrm{Da}=10^{-3}$
In addition, the increase in porosity in the channel makes the velocity field larger and therefore the intensification of the flow. Therefore, the high porosity facilitates the flow of fluid through the porous layers.

The observations made previously can be confirmed from the temperature profiles in Figure 3 below for different porosities. This figure illustrates that the increase in porosity is followed by a decrease in the overall temperature field. So, the heat transfer in the channel is amplified for low porosities.

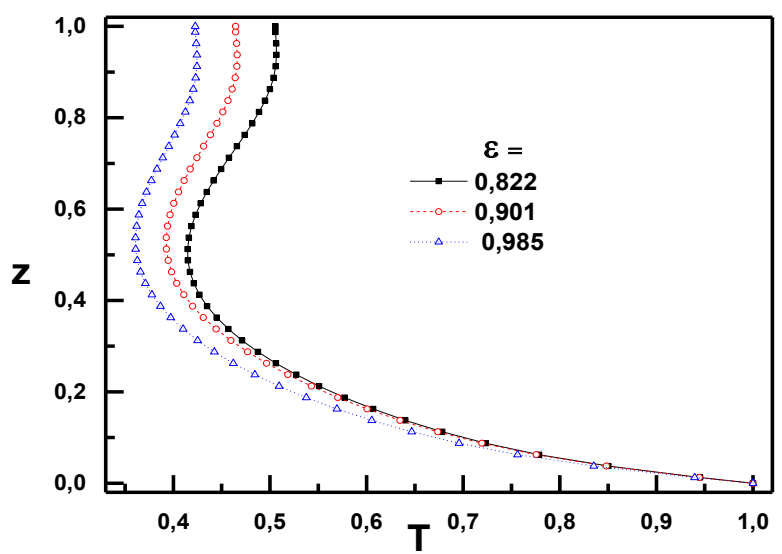

Fig. 3. Temperature profiles for different porosities, $\operatorname{Re}=50$, $\mathrm{Ra}=10^{4},(\tilde{\lambda}=1)$ and $\mathrm{Da}=10^{-3}$

\subsection{Effect of Rayleigh Number}

Figure 4 below defines the evolution of the global Nusselt number $\left(\mathrm{Nu}_{\mathrm{g}}\right)$ as a function of the Rayleigh number $\left(\mathrm{Ra}=100\right.$ to $\left.\mathrm{Ra}=5.10^{5}\right)$ for $\mathrm{Re}=50, \mathrm{Da}=10^{-3}$ and for different porosities $(\varepsilon)$. The effects of the Rayleigh number ( $\mathrm{Ra}$ ) on heat transfer are obvious, we can see that the increase in the number of Rayleigh ( $\mathrm{Ra})$ is followed by an increase in the Nussselt number $\left(\mathrm{Nu}_{\mathrm{g}}\right)$. Indeed, for a porosity $\varepsilon=0.822$, the heat exchange is more significant than in the case of a higher porosity $(\varepsilon=$ $0.975)$, moreover the decrease of the porosity $(\varepsilon)$ causes a slowing down of the flow in the foam and subsequently an increase in the heat exchange between the solid matrix and the fluid which explains the increase of the heat transfer in the cavity.

\subsection{Thermal conductivity ratio effect}

Nusselt number: This part is devoted to the study of the thermal interactions between the solid matrix and the fluid phase. We will therefore study the effect of the ratio of thermal conductivities $(1 \leq \tilde{\lambda} \leq 700)$ for two different porosities 0.822 and 0.97 by fixing the other parameters governing our problem such as $\mathrm{Ra}=10^{4}$, $\mathrm{Re}=50, \mathrm{Da}=10^{-3}$. The evolution of the Nusselt number, reflecting the heat transfer, with respect to the thermal conductivities ratio $(\tilde{\lambda})$ is illustrated in Figure 5. 


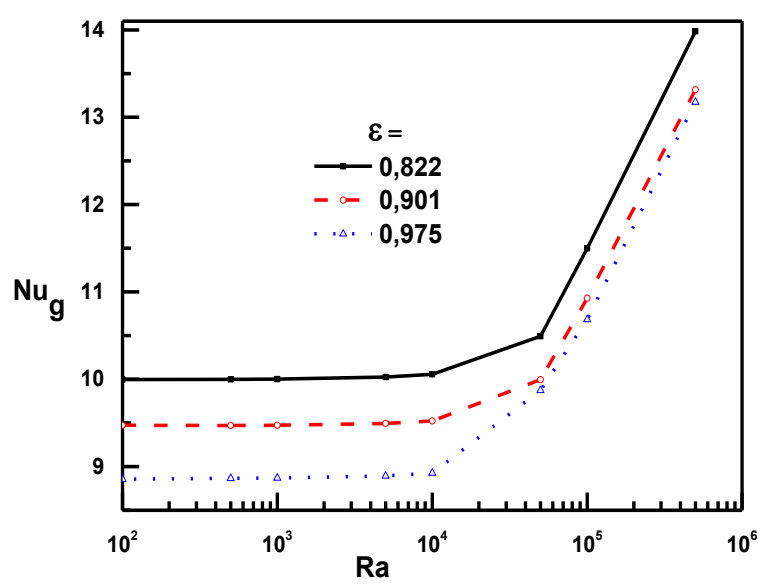

Fig. 4. Evolution of the global Nusselt number $(\mathrm{Nug})$ as a function of $(\mathrm{Ra})$ for different porosities, $\mathrm{Da}=10^{-3}, \mathrm{Re}=50$ and $(\tilde{\lambda}=1)$.

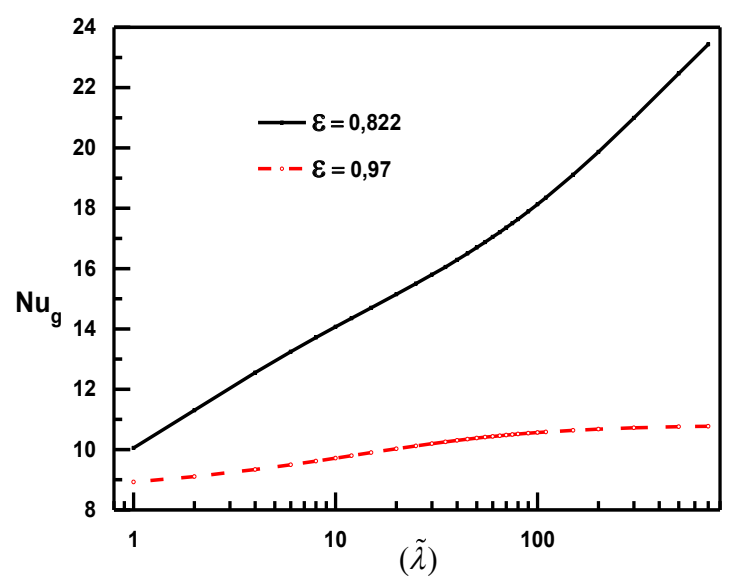

Fig. 5. Evolution the global Nusselt number as a function of thermal conductivity ratio $(\tilde{\lambda})$ for different porosities for $\mathrm{Ra}=10^{4}, \mathrm{Re}=50$ and $\mathrm{Da}=10^{-3}$

This figure reveals that the global Nusselt number $\left(\mathrm{Nu}_{\mathrm{g}}\right)$ increases rapidly with the increase of the thermal conductivity $\operatorname{ratio}(\tilde{\lambda})$ for porosity $\varepsilon=0.822$. Which proves that the heat transfer is amplified for the high values of $(\tilde{\lambda})$ and therefore the transfer mode is almost conductive imposed by the thermal conductivity of the solid matrix of the metal foam. Whereas for a high porosity $\varepsilon=0.97$, there is a slight increase in the Nusselt number (Nug) for $(\tilde{\lambda}=10)$, then the evolution of the global Nusselt number(Nug) reaches its maximum and remains constant with a horizontal asymptotic pace, which explains the increase in conductive heat transfer.

Isotherms and streamtraces: The effect of the thermal conductivity ratio $(\tilde{\lambda})$, where it's varied from 10 to 700 on the isotherms velocity field and streamtraces are displayed in figure 6 .
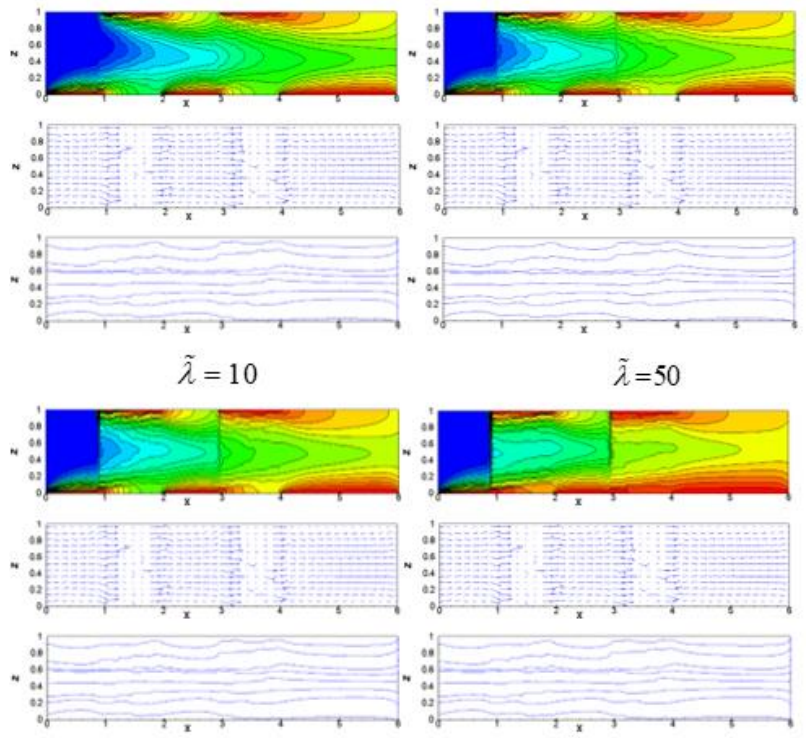

$\tilde{\lambda}=100$

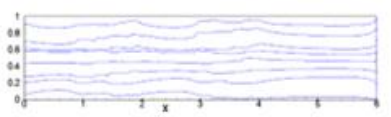

$\tilde{\lambda}=700$

Fig. 6. Isotherms, velocity fields and streamtraces for different $(\tilde{\lambda}), \operatorname{Re}=50, \mathrm{Ra}=10^{4}, \varepsilon=0.822$ and $\mathrm{Da}=10^{3}$

Velocity fields and streamtraces show a rehomogenization of the flow in the porous matrix due to the low Darcy number of porous blocks $\left(\mathrm{Da}=10^{-3}\right)$ which slows the movement of the fluid. The isotherms illustrate the important effect of thermal interactions between porous media and fluid. Indeed, the increase in $(\tilde{\lambda})$ leads to the intensification of the porous blocks and the isotherms are more dispersed in the porous matrix. For the low thermal conductivity ratios $(\tilde{\lambda}=10)$, the isotherms are quite curved with low temperature gradients, hence the movement of the fluid is very convective. Whereas, starting from $(\tilde{\lambda}=50)$, there is a thermal quasi-stratification which explains the gradual attenuation of the convective transfer giving rise to the conductive transfer. As a result, metallic foam with its very high thermal conductivity leads to an increased heat transfer by conduction through the solid matrix.

\subsection{Effect of porous number blocks}

Figure 7 shows the temperature profiles at outlet of the channel for different numbers of metal foam blocks for $\mathrm{Re}=50, \mathrm{Ra}=10^{4}, \mathrm{Da}=10^{-3},(\tilde{\lambda}=1)$ and $\varepsilon=0.822$. It is found that the number of metal foam blocks is a parameter of optimal conditions control in order to improve the heat transfer in the cavity. Indeed, the best choice is to use two blocks of metal foam since the temperature profiles for different planes $(\mathrm{y}=0.1,0.5$ and $0.9)$ are almost identical displaying the best thermal mixing. 


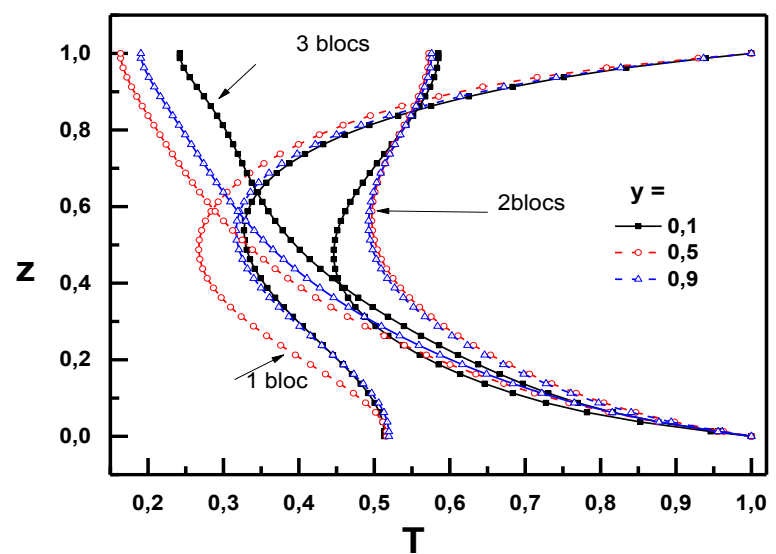

Fig. 7. Temperature profiles at the outlet of the cavity for different numbers of metal foam blocks for $\mathrm{Re}=50, \mathrm{Ra}=10^{4}$,

$$
\mathrm{Da}=10^{-3},(\tilde{\lambda}=1) \text { and } \varepsilon=0.822
$$

\section{Conclusion}

In this paper we have illustrated the results for mixed convection in a in a channel partially filled with metal foam block and solicited by solar radiation out of local thermal equilibrium.

The results of our simulation allow us to conclude that:

- The increase in the Rayleigh number generates the increase in heat transfer and the intensification of the flow which reveals the transition from a conductive regime to that totally convective.

- The increase in porosity (increase in the permeability of the metallic foam) causes the intensification of the flow in the channel, while the heat transfer is amplified for low porosities

- For high thermal conductivity ratios, heat transfer is amplified and the homogenization of the temperature at the outlet of the channel is ensured.

- The choice of two metal foam blocks is the optimal number of blocks which revealed temperature profiles show the best thermal mixing

\section{References}

1. A. Bejan and D.A. Nield, Conv in Porous Media, (Springer, New York, 2013).

2. K. Vafai, Handbook of Porous Media, (New York, 2005)

3. M. Kaviany, Principles of Heat Transfer in Porous Media, (Springer-Verlag, New York, 1991)

4. F. Habbachi, F.S. Oueslati, M. Ganaoui, R. Bennacer and A. Elcafsi, Energy Procedia, 139, 617-623, (2017).

5. A. Kacem, F.S. Oueslati, R. Bennacer and A. Elcafci, Def. Diffus. Forum 348, 27 (2014).

6. W. Lu, T. Zhang and M. Yang, Int.J. Heat Mass Transf. 100, 162 (2016)

7. B. Buonomo, A. Diana, O. Manca and S. Nardini, J. Phys. Conf. Ser. 923 (2017)
8. B. Buonomo, G. Ferraro, O. Manca, L. Marinelli and S. Nardini, J. Phys. Conf. Ser. 501 (2014).

9. F.S. Anuar, I.A. Abdi and K. Hooman, Int.J. Heat Mass Transf. 127, 1197 (2018)

10. X. Bai, F. Kuwahara, M. Mobedi and A. Nakayama, J. Heat Transf. 140 (2018)

11. S. V. Patankar, Numerical heat transfer and fluid flow, New York, (1980) 